\title{
Síndrome de Burnout em Professores Moçambicanos do Ensino Fundamental
}

\author{
Gildo Aliante \\ ${ }^{1}$ Universidade Federal do Rio Grande do Sul, RS, Brasil. \\ Jaqueline Tittoni ${ }^{1}$ \\ ${ }^{1}$ Universidade Federal do Rio Grande do Sul, RS, Brasil.
}

\author{
Mary Sandra Carlotto ${ }^{2}$ \\ ${ }^{2}$ Universidade do Vale do Rio dos Sinos, RS, Brasil. \\ Mussa Abacar ${ }^{3}$ \\ ${ }^{3}$ Universidade Rovuma, Nampula, Moçambique.
}

Resumo: A docência figura na lista das profissões mais acometidas pela síndrome de burnout (SB) devido à presença de uma multiplicidade de fatores. $\mathrm{O}$ objetivo desta pesquisa foi avaliar a frequência da síndrome em professores moçambicanos do ensino fundamental, verificando as possíveis associações com as variáveis sociodemográficas e laborais. A pesquisa de natureza quantitativa possui um delineamento observacional, analítico, transversal e envolveu 263 professores, sendo $56,3 \%$ do sexo feminino, com idades variadas entre 18 e 58 anos. A coleta de dados foi feita por meio de questionário de dados sociodemográficos e laborais do "Cuestionario para la evaluación del síndrome de quemarse por el trabajo", versão em português para profissionais de educação (CESQT-PE). Os dados foram analisados no software SPSS (Statistical Package for Social Sciences). Os resultados indicam que 13,3\% dos participantes apresentaram baixos níveis em ilusão pelo trabalho, 16,3\% altos níveis em desgaste psíquico, 13,3\% em indolência e 25,9\% altos níveis de culpa. Na análise de perfis, $24,0 \%$ enquadram-se no perfil 1 (altos níveis da SB) e 11,0\% no perfil 2 (níveis severos da SB). Verificou-se que houve associação estatisticamente significativa apenas entre a variável "idade" com a dimensão de desgaste psíquico e da variável "sexo" com a dimensão de culpa. Conclui-se que alguns professores estão em fase de desenvolvimento da síndrome, necessitando de intervenções para reverter o cenário. Esses resultados chamam a atenção dos gestores e da comunidade escolar para a necessidade de melhorar os fatores geradores de estresse ocupacional, de modo a transformar o trabalho docente em saudável e prazeroso.

Palavras-chave: Saúde do Trabalhador, Saúde Mental, Síndrome de Burnout.

\section{Burnout Syndrome in Mozambican Teachers of Elementary School}

\begin{abstract}
Due to the multiplicity of stressors inherent to teaching, this occupation is one of the most affected by the burnout syndrome. This research sought to evaluate the frequency of Burnout among elementary schoolteachers in Mozambique, verifying possible associations between this condition and sociodemographic and occupational factors. This is a quantitative study, with an observational, analytical, transversal design, conducted with 263 teachers $-56.3 \%$ of whom were female and age from 18 to 58 years. Sociodemographic and occupational data were collected using the Portuguese version of the Spanish Burnout Inventory for Educational Professionals (CESQT-PE) and analyzed using the Statistical Package for the Social Sciences Software. The results indicate that $13.3 \%$ of the participants had low levels of illusion for work, $16.3 \%$ had high levels of mental deterioration, $13.3 \%$ had indolence, and $25.9 \%$ had high levels of guilt. Profile analysis showed that $24 \%$ of the evaluated teachers fit into profile 1 (high levels of burnout) and $11 \%$ into profile 2 (severe levels of burnout). Age was significantly associated with mental deterioration and gender with guilt. These findings indicate that some teachers are developing the burnout syndrome, thus requiring interventions to prevent the worsening of
\end{abstract}


such condition. This study draws the awareness of managers and the school community to the need for improving factors that generate occupational stress, aiming to transform the teaching work into a healthy and pleasant task.

Keywords: Worker's Health, Mental Health, Burnout Syndrome.

\title{
Síndrome de Quemarse por el Trabajo en Profesores Mozambiqueños de la Primaria
}

\begin{abstract}
Resumen: La docencia está entre las profesiones más acometidas por el síndrome de quemarse por el trabajo (SQT) debido a múltiples factores. El objetivo de esta investigación fue evaluar la frecuencia del síndrome de quemarse por el trabajo en profesores mozambiqueños de la primaria, verificando posibles asociaciones con las variables sociodemográficas y laborales. Esta es una investigación cuantitativa, de tipo observacional, analítica, transversal, en la cual participó 263 profesores, siendo el 56,3\% del sexo femenino, con edades de entre 18 y 58 años. Para recopilar los datos se aplicó el cuestionario de datos demográficos y de empleo, y el Cuestionario para la Evaluación del Síndrome de Quemarse por el Trabajo para Profesionales de la Educación (CESQT-PE), versión en portugués. Los datos se analizaron con el uso del software Statistical Package for Social Sciences (SPSS). Los resultados indican que el 13,3\% de los participantes presentaron bajos niveles en ilusión por el trabajo; el 16,3\%, altos niveles en desgaste psíquico; el 13,3\% en indolencia; y el 25,9\% altos niveles de culpa. En el análisis de perfiles, el 24,0\% se encuadran en el perfil 1 (altos niveles de SQT) y el 11,0\% en el perfil 2 (niveles severos de SQT). Se verificó que solo hubo asociación estadísticamente significativa entre la variable "edad" con la dimensión desgaste psíquico y la variable "sexo" con la dimensión culpa. Se concluye que algunos profesores se encuentran en fase de desarrollo del síndrome, necesitando intervenciones para tratar lo tempranamente. Estos resultados llaman la atención de los gerentes y la comunidad escolar sobre la necesidad de mejorar los factores que generan estrés ocupacional para volver el trabajo docente saludable y placentero.
\end{abstract}

Palabras clave: Salud del Trabajador, Salud Mental, Síndrome de Quemarse por el Trabajo.

\section{Introdução}

As diversas mudanças e transformações que ocorrem no contexto da organização escolar aumentam cada vez mais a complexidade da profissão docente (Vasconcelos \& Neves, 2010). Essas mudanças agravaram-se na realidade moçambicana principalmente a partir do ano de 2004, quando teve início a implementação das reformas curriculares em todas as escolas do ensino básico. Tais inovações abrangeram a introdução de ciclos de aprendizagem; o ensino básico integrado; o currículo local; a distribuição de professores para cada classe do ensino primário do segundo grau; a progressão por ciclos de aprendizagem, a introdução de línguas moçambicanas e das disciplinas de educação moral e cívica, inglês, ofícios, educação musical e línguas nacionais (INDE/MINED, 2003).
Houve igualmente o aumento da carga horária do professor em termos qualitativos e quantitativos, ou seja, o número de disciplinas por lecionar passou de três para oito na $1^{\text {a }}$ e $2^{\text {a }}$ classe, de quatro para oito na $3^{\text {a }}$ classe e de cinco para nove nas $4^{\mathrm{a}}$ e $5^{\mathrm{a}}$ classes, respectivamente.

No ensino primário do segundo grau (EP2) $\left(6^{\mathrm{a}} \mathrm{e}\right.$ $7^{\text {a }}$ classe), o número de professores para cada classe foi reduzido de sete para três ou quatro. O INDE/ MINED (2003), organismo que subentende a área de educação em Moçambique, fundamentou a redução dos professores nos seguintes termos:

A redução do número de docentes por turma no EP2, de sete, para três, tem, como pano de fundo, a organização do currículo em áreas disciplinares. A presente opção tem em vista uma rápida expan- 
são da rede do EP2, a nível nacional, visto que o sistema de três professores para o EP2 se afigura menos dispendioso (INDE/MINED, 2003, p. 28).

É importante observar ainda que a implementação das inovações raramente foi acompanhada pela capacitação dos professores, provisão de recursos materiais e melhoria das condições de trabalho, o que possivelmente vem gerando insatisfação e desmotivação profissional e, por conseguinte, uma queda significativa na qualidade de aprendizagem do ensino público em Moçambique (MINED, 2015). É nesse contexto que pesquisadores tecem críticas contundentes sobre a qualidade de ensino e aprendizagem dos alunos nas escolas moçambicanas (Abacar, Roazzi, \& Bueno, 2017). Em face disso, Cruz, Lemos, Welter e Guisso (2010) esclarecem que as condições e as múltiplas exigências feitas ao professor têm sido cada vez mais associadas aos problemas de saúde física e mental apresentados pelos docentes, tais como a fadiga psicológica, o estresse e a síndrome de burnout (SB), aspectos que podem comprometer o bem-estar, a longevidade na profissão e a qualidade de suas interações com os alunos (Lhospital \& Gregory, 2009).

A SB é, de acordo com Maslach e Jackson (1981) e Schaufeli e Enzamann (1998), uma resposta prolongada aos estressores interpessoais crônicos ao trabalho que acomete profissionais que mantêm relação constante e direta com outras pessoas, como professores, médicos, enfermeiros, psicólogos, assistentes sociais, policiais, bombeiros, dentre outros. O modelo proposto por Maslach e Jackson (1981), Maslach e Goldberg (1998), Maslach e Leiter (2017) é descrito em três fases: a) exaustão emocional: o trabalhador, depois de uma interação intensiva com os clientes, denota desgaste de suas energias emocionais e adverte que não pode trabalhar com a mesma dedicação e energia que apresentava no princípio de sua carreira profissional. Essa dimensão manifesta-se por meio do esgotamento de recursos emocionais próprios, isto é, o profissional sente que não pode dar mais de si mesmo em nível emocional; b) despersonalização: manifesta-se por atitudes negativas, como o tratamento depreciativo, atitudes frias e distantes e/ou desconexão dos problemas dos clientes. Essa dimensão pode entender-se como um modo de enfrentamento à exaustão emocional que o trabalhador experimenta e; c) baixa realização pessoal no trabalho: traduz-se em uma valoração negativa do papel profissional. O trabalhador, desgastado profissionalmente, sente-se insatisfeito com seu trabalho, o que o leva a revelar sentimentos de ineficácia no desenvolvimento de suas tarefas.

Por sua vez, Gil-Monte (2005) elaborou um modelo teórico que caracteriza a SB em quatro dimensões: ilusão pelo trabalho, desgaste psíquico, indolência e culpa. Esse modelo considera a SB como uma resposta ao estresse laboral crônico, característico dos profissionais que trabalham no atendimento de pessoas. Nele, as deteriorações cognitivas (baixas pontuações na ilusão pelo trabalho) e afetivas (altas pontuações em desgaste psíquico) aparecem inicialmente como respostas às fontes de estresse laboral crônico e, consequentemente, os indivíduos passam a desenvolver atitudes negativas frente às pessoas que atendem no trabalho (altos níveis de indolência).

O surgimento do sentimento de culpa é posterior a esses sintomas, mas não ocorre necessariamente em todos os indivíduos (Gil-Monte, Carlotto, \& Câmara, 2010). Esse é o modelo adotado para este estudo, tendo em vista que o instrumento usado para a coleta de dados considera a SB nessa perspectiva. De modo geral, a síndrome pode ter origem na cronicidade de demandas do ambiente do trabalho, tais como: conflito e ambiguidade de papéis, sobrecarga do trabalho, injustiças, falta de participação no processo decisório, falta de apoio e suporte social e profissional, conflitos interpessoais, baixa remuneração, assédio e conflito trabalho-família (Lambert, Hogan, Griffin, \& Kelley, 2015).

No ambiente educacional (i.e. instituições de ensino), diversos estressores ocupacionais da docência podem levar à SB. Nesse contexto, Olaitan Oyerinde, Obiyemi e Kayode (2010) verificaram que más relações interpessoais com colegas, currículo centralmente desenhado, falta de participação dos pais na educação, cobranças advindas da sociedade, fraca aprendizagem dos alunos, autoridade escolar, ambiente de ensino impróprio e baixos salários eram as principais fontes de estresse relatadas pelos professores nigerianos.

No estudo de Genoud, Brodard e Reicherts (2009), a indisciplina dos alunos, a carga administrativa, os relacionamentos negativos com as autoridades, os maus relacionamentos com colegas e relacionamentos ruins com pais foram os principais fatores associados à SB em professores suíços. Em Portugal, Pocinho e Capelo (2009) identificaram os seguintes estressores 
ocupacionais dos professores: comportamentos inadequados e indisciplina dos alunos, pressões de tempo e excesso de trabalho. Na mesma direção, Gomes, Peixoto, Pacheco e Silva(2012) constataram que comportamentos inadequados e indisciplina dos alunos, pressões de tempo, excesso de trabalho, trabalho burocrático e administrativo, políticas disciplinares inadequadas, diferentes capacidades e motivações dos alunos foram as principais fontes geradoras de estresse ocupacional em professores portugueses.

A pesquisa de Antoniou, Ploumpi e Ntalla (2013), envolvendo uma amostra de professores gregos, revelou a sobrecarga de tarefas, as condições de trabalho, o desinteresse e desmotivação dos estudantes e a falta de apoio do governo como os principais aspectos negativos e estressores da profissão. Na África do Sul, o estudo de Zhuwao, Setati, Rachidi e Ukpere (2015) também demonstrou que as características do trabalho, sobrecarga, pouca participação na tomada de decisões e a relação profissional constituem os principais estressores ocupacionais dos professores.

No Brasil, Carlotto et al. (2018) identificaram cinco estressores de natureza psicossocial em professores: má relação interpessoal entre professor-alunos, falta de apoio de familiares de alunos, sobrecarga de papel, dificuldades de conciliar trabalho-família e trabalho-vida pessoal. Similarmente, a pesquisa de Oliveira, Balk, Graup e Muniz (2020) mostrou os seguintes fatores que influenciam na degradação do estado de saúde mental de professores brasileiros: desvalorização profissional, baixos salários e mau relacionamento interpessoal.

Estudos realizados no contexto moçambicano sugerem a existência de vários fatores que contribuem para a ocorrência de estresse em professores do ensino básico e que podem gerar a SB nessa classe profissional. Um estudo comparado entre professores brasileiros e moçambicanos do ensino básico, realizado por Abacar et al. (2017), apontou os baixos salários e benefícios, o difícil relacionamento com os pais e encarregados da educação, a limitação no desenvolvimento de carreira, a sobrecarga de trabalho, a pressão de tempo, a insuficiência e inadequação de recursos materiais e físicos como principais fontes de estresse no trabalho docente. Resultados similares foram encontrados por Aliante e Tittoni (2017) entre professores do ensino básico.

De modo semelhante, Aliante (2018) apontou inúmeros fatores de estresse do professor, incluindo os encontrados em pesquisas anteriores, nomeadamente: falta de atribuição de bolsas para continuação de estudo; atribuição da culpa pela fraca aprendizagem dos alunos; falta de recursos materiais para o trabalho; baixos salários; viver longe da família; falta de apoio social; inexistência de planos de benefícios sociais; falta de datas fixas de pagamento de salários; mudanças ou atualizações regulares de currículos; quantidade de alunos por turma; distância percorrida entre escola-casa e vice-versa e precárias condições físicas e materiais de trabalho. Estudos recentes de Abacar, Aliante e Nahia (2020) e Aliante, Abacar e Pereira (2020) evidenciaram os seguintes fatores do estresse na profissão docente: precárias condições de trabalho, salários baixos, falta de desenvolvimento profissional, políticas de educação e sobrecarga de trabalho, maior quantidade de alunos por turma, comportamento dos alunos e fraca aprendizagem, deficiente participação dos pais e/ou encarregados da educação.

Em Moçambique, além dos riscos do trabalho do professor e da precariedade das condições em que a prática docente se realiza, há insatisfação dos professores do ensino básico da rede pública em relação às suas expectativas sociais e à perspectiva de crescimento profissional, o que pode gerar problemas de saúde psíquica, ou seja, favorecer a ocorrência de índices de estresse no trabalho (Abacar, 2011, Abacar et al., 2020; Aliante, et al., 2020) e da SB (Abacar, 2015; Abacar et al., 2020; Abacar, Tarcísio, \& Aliante, 2017). Possivelmente, um maior número de professores moçambicanos, particularmente do ensino básico, pode estar acometido pela SB. No entanto, devido ao desconhecimento da síndrome, os professores e os órgãos de gestão não têm consciência sobre como a doença se manifesta, suas causas e efeitos (Abacar, 2015), apesar das enormes consequências tanto para a saúde do trabalhador quanto para o funcionamento das organizações.

A revisão da literatura feita por Salvagioni et al. (2017), que resume as evidências das consequências físicas, psicológicas e ocupacionais da SB, concluiu que esse fenômeno é um preditor significativo das seguintes consequências físicas: hipercolesterolemia, diabetes, doença de insuficiência cardíaca, doença cardiovascular, dor musculoesquelética, alterações nas experiências de dor, fadiga prolongada, dores de cabeça, problemas gastrointestinais, problemas respiratórios, lesões graves e mortalidade. Os efeitos psicológicos são insônia, sintomas depressivos, 
uso de medicações psicotrópicas e antidepressivas, hospitalização por transtornos mentais, insatisfação no trabalho, absenteísmo, nova aposentadoria por invalidez, demanda por trabalho, recursos para o trabalho e presenteísmo.

No caso particular dos profissionais da educação, a SB também afeta o ambiente educacional e interfere na obtenção dos objetivos pedagógicos, levando-os a um processo de alienação, cinismo, apatia, problemas de saúde e intenção de abandonar a profissão (Guglielmi \& Tatrow, 1998). Concomitantemente, Youg e Yue (2007) alertam que a SB afeta gravemente a saúde física e mental dos professores, reduzindo a qualidade de seu trabalho e, consequentemente, prejudica a saúde física e mental de alunos, comprometendo o desenvolvimento saudável da educação.

Embora a SB seja um problema de caráter epidêmico mundial que afeta profissionais de diferentes países, despertando maior atenção nos pesquisadores (Gil-Monte, 2008), e haja uma série de estudos empíricos sobre o fenômeno em professores de diferentes tipos e níveis de ensino, há pouca sensibilização e pesquisas a respeito do assunto em países do continente africano (Amimo, 2012), particularmente entre países em desenvolvimento (Badawi, 2015), como é o caso de Moçambique. Além da escassa existência de estudos sobre a SB em professores moçambicanos, os existentes até então examinaram a síndrome aplicando o Maslach Burnout Inventory (MBI) como instrumento, não havendo qualquer estudo que utilizou o "Cuestionario para la evaluación del síndrome de quemarse por el trabajo", versão em português para profissionais de educação (CESQT-PE). Assim, este estudo amplia o modelo de mensuração da SB por meio de CESQT-PE, uma medida alternativa do MBI que tem sido recorrentemente utilizada em diferentes países, principalmente de língua não inglesa (GilMonte et al., 2010).

Com base no referencial teórico apresentado, esta pesquisa busca responder ao problema central expresso nos seguintes termos: qual é a frequência da SB em professores moçambicanos do ensino básico da rede pública na cidade de Nampula, Moçambique, e que variáveis sociodemográficas e laborais estão associadas?

\section{Metodologia}

A metodologia de referência para este estudo está explicitada nesta sessão. Inicialmente, identifica-se o desenho de pesquisa. A seguir, descrevem-se os instrumentos de coleta de dados, os procedimentos e as estratégias para análise de dados.

\section{Tipo de pesquisa}

Esta pesquisa é de natureza quantitativa, pois recorre aos instrumentos de mensuração e técnicas estatísticas para a coleta, classificação e análise dos dados, e tem delineamento observacional, analítico e transversal. Trata-se de um estudo descritivo e analítico pelo fato de verificar as associações entre as variáveis sociodemográficas e laborais (e.g., sexo, idade, estado cível, número de filhos, nível de escolarização, número de alunos atendidos, carreira/categoria, anos de experiência profissional) e as dimensões da SB.

\section{Instrumentos de coleta de dados}

A coleta de dados foi realizada por meio dos seguintes instrumentos padronizados e autoaplicáveis: questionário sobre dados sociodemográficos e laborais e Cuestionario para la evaluación del síndrome de quemarse por el trabajo, versão em português para profissionais de educação (CESQT-PE), adaptada para o português do Brasil (Gil-Monte et al., 2010) e de Portugal (Figueiredo-Ferraz, Gil-Monte, \& Grau-Alberola, 2013). O questionário de dados sociodemográficos levantou informações pessoais e laborais, como idade, sexo, estado civil, tempo de serviço, carreira/categoria, nível de escolarização, número de alunos e carga horária semanal.

O CESQT-PE possui 20 itens distribuídos em quatro subescalas: ilusão pelo trabalho (5 itens), desgaste psíquico ( 4 itens), indolência ( 6 itens) e culpa (5 itens). $\mathrm{O}$ instrumento apresenta frases afirmativas expressando sentimentos de diversas ordens, em que cada respondente marca apenas uma opção na frase conforme a classificação: 0 - nunca, 1 - raramente ou algumas vezes por ano, 2 - às vezes: algumas vezes por mês, 3 - frequentemente: algumas vezes por semana e 4 - muito frequente ou todos os dias, de acordo com a sua situação específica.

A estrutura interna do instrumento é a seguinte: a) ilusão pelo trabalho: indica o desejo individual para atingir metas relacionadas ao trabalho, percebidas pelo sujeito como atraentes e fonte de satisfação pessoal (5 itens; $\alpha=0,83$ ); b) desgaste psíquico: caracterizado pelo sentimento de exaustão emocional e física em relação ao contato direto com pessoas que são fontes ou causadoras de problemas ( 4 itens; $\alpha=0,80$ ); c) indolência: 
manifestada por meio de atitudes de indiferença junto às pessoas que necessitam ser atendidas no ambiente de trabalho, assim como insensibilidade aos problemas alheios ( 6 itens; $\alpha=0,80$ ); e d) culpa: evidenciada pelo surgimento de cobrança social e sentimento de culpabilização relativa ao comportamento e atitudes negativas desenvolvidas no trabalho, sendo evidenciada especialmente em sujeitos que desenvolvem relações diretas no ambiente de trabalho ( 4 itens; $\alpha=0,82$ ).

A avaliação do CESQT-PE permite determinar dois tipos de perfis. Aqueles que apresentam simultaneamente baixas pontuações em ilusão pelo trabalho e altas pontuações em desgaste psíquico e indolência representam altos níveis da SB e são atribuídos ao perfil 1. No caso de sujeitos do perfil 2, as altas pontuações são ainda acompanhadas de altos níveis na dimensão culpa e são considerados sujeitos com níveis severos da SB (Figueiredo-Ferraz et al., 2013; Gil-Monte, 2005; Gil-Monte et al., 2010).

\section{Procedimentos}

Esta pesquisa foi desenvolvida nas escolas do ensino primário (i.e. fundamental) localizadas na cidade de Nampula, em Moçambique. A coleta de dados foi realizada nos meses de fevereiro a junho de 2018. Primeiramente, foram efetuados contatos com o Serviço Distrital de Educação, Juventude e Tecnologia de Nampula (SDEJTN) por meio de carta que apresentava o objetivo da investigação e solicitava autorização para a sua realização. Após a autorização (N/Ref. no 254/ SDEJTN/SG/010.2/2018, datado em 28 de fevereiro de 2018), foi passada a credencial para livre acesso às escolas.

O projeto de pesquisa foi submetido ao Comitê de Ética e Pesquisa (CEP) do Instituto de Psicologia da Universidade Federal do Rio Grande do Sul (Ufrgs), e, por ser realizada em Moçambique, o CEP orientou que a pesquisa deveria seguir as normas vigentes naquele país. No entanto, devido à impossibilidade de encontrarmos um comitê dessa natureza em Nampula, foi solicitada a autorização por parte do órgão responsável pela gestão do Sector da Educação na cidade de Nampula (SDEJTN), e os pesquisados assinaram o termo de consentimento livre e esclarecido elaborado pelos pesquisadores. O material da pesquisa segue sob os cuidados da pesquisadora responsável (a segunda autora) no Instituto de Psicologia da Ufrgs.

Posteriormente, as direções das escolas e os professores foram contatados e informados sobre os objetivos do estudo e, finalmente, responderam individualmente aos questionários de pesquisa, mediante instruções dadas pelo primeiro autor do trabalho. Alguns questionários foram aplicados nas salas de aulas da Universidade Pedagógica, Delegação de Nampula (atual Universidade Rovuma), onde estudava uma parte de professores que lecionam em diversas escolas localizadas na cidade de Nampula. A aplicação dos questionários naquela instituição de ensino superior teve autorização oral da chefe do departamento de Ciências de Educação e Psicologia. A participação dos professores na pesquisa foi de livre e espontânea vontade, e foi garantido e mantido o anonimato dos dados.

\section{Análise de dados}

A análise de dados foi feita por meio do programa Statistical Package for Social Sciences (SPSS, versão 22.0). Elaborou-se análises estatísticas descritivas (média e desvio-padrão) para o levantamento dos dados do questionário sociodemográfico e laboral. A frequência da SB foi analisada segundo o procedimento dos pontos de referência da escala de frequência de respostas, tendo o ponto de corte de 2 (algumas vezes ao mês). Foi realizado o teste de independência Qui-quadrado de Pearson (nível de significância de 5\%) para dados categóricos, com objetivo de verificar a associação entre as variáveis sociodemográficas $\mathrm{e}$ laborais qualitativas e os níveis das dimensões da SB. Igualmente, fez-se os testes de correlação de Spearman devido a não uniformidade dos dados, para verificar a associação entre as quatro dimensões da SB e as variáveis sociodemográficas e laborais quantitativas.

\section{Resultados e discussão}

A análise e a discussão dos resultados deste estudo são apresentadas nesta sessão. Inicialmente, mostra-se a caracterização da amostra do estudo e, posteriormente, são apresentados e discutidos os resultados da pesquisa em função das categorias de análise, a saber, frequência da síndrome de burnout, determinação dos perfis da SB, correlação das dimensões da síndrome com as variáveis sociodemográficas qualitativas e quantitativas.

\section{Perfis dos participantes}

A pesquisa envolveu 263 professores e professoras, sendo 115 (43,7\%) do sexo masculino e 148 (56,3\%) do sexo feminino. A média de idade dos participantes era de 33,2 anos (DP =6,7). Em termos de estado civil, 
198 (75,3\%) participantes eram casados e/ ou união de fato, $55(20,9 \%)$ solteiros, seis $(2,3 \%)$ viviam separados e quatro $(1,5 \%)$ se declararam como viúvos. Quanto à escolaridade, $216(81,1 \%)$ tinham nível médio, $24(9,1 \%)$ ensino superior e $23(8,8 \%)$ nível básico. A média do número de filhos dos professores foi de três ( $\mathrm{DP}=1,8$ ) numa amplitude de 0-8 filhos.

A maioria dos participantes, 239 (90,9\%), tinha vínculo profissional estável (nomeação), e 24 (9,1\%) eram contratados. Em termos de enquadramento na carreira/categoria, 105 (39,9\%) estavam na docência do nível quatro (DN4), ou seja, básicos; 147 (55,9\%) do nível três (DN3), istoé, médios profissionais; e 11 (4,2\%) eram do nível superior (um docente do nível dois DN2 e 10 do nível um - DN1). A média dos alunos para cada professor estimou-se em $87(\mathrm{DP}=66,8)$. Quanto aos anos de experiência profissional, a média era de $10(\mathrm{DP}=5,5)$. A carga horária semanal média foi de 25 aulas $(\mathrm{DP}=3,4)$. Predominaram participantes formados no modelo de magistério de $10+1$ ano, sendo 126 professores $(47,9 \%)$, seguido de $10+2$ anos com 79 $(30,0 \%), 28(10,7 \%)$ do modelo $7+3$ anos, $13(4,9 \%)$ do modelo $10+3$ anos, $11(4,2 \%)$ são do $12+1$ ano, e seis $(2,3 \%)$ tinham formação superior.

\section{Médias das dimensões e perfis da síndrome de burnout}

Com objetivo de avaliar a frequência da SB nos professores estudados, foram calculados as médias e os desvios-padrão para cada dimensão, com base na frequência das respostas dos participantes, como ilustra a Tabela 1.

\section{Tabela 1}

Frequência da síndrome de burnout entre professores participantes do estudo.

\begin{tabular}{lcccc}
\hline \multicolumn{1}{c}{ Subescalas } & M & DP & Níveis baixos $(<2)$ & Níveis altos $(\geq \mathbf{2})$ \\
\hline Ilusão pelo trabalho & 2,84 & 0,82 & $35(13,3 \%)$ & $228(86,7 \%)$ \\
Desgaste psíquico & 0,93 & 0,86 & $220(83,7 \%)$ & $43(16,3 \%)$ \\
Indolência & 1,16 & 0,65 & $228(86,7 \%)$ & $35(13,3 \%)$ \\
Culpa & 1,31 & 0,84 & $195(74,1 \%)$ & $68(25,9 \%)$ \\
Perfil 1 (15 itens) & 1,64 & 0,50 & $200(76 \%)$ & $63(24 \%)$ \\
Perfil 2 (P1 + Culpa) & 1,56 & 0,50 & $234(89 \%)$ & $29(11 \%)$ \\
\hline
\end{tabular}

De acordo com a Tabela 1, a dimensão que obteve maior média foi da ilusão pelo trabalho, com 2,84 (DP = 0,82), seguida da dimensão de culpa, com 1,31 ( $\mathrm{DP}=0,84)$, e as dimensões de indolência e desgaste psíquico obtiveram as médias de $1,16$ (DP $=0,56)$ e 0,93 (DP = 0,86) respectivamente.

A frequência da SB foi avaliada a partir do procedimento dos pontos de referência da escala de frequência de respostas. Desse modo, 35 participantes $(13,3 \%)$ apresentaram baixos níveis em ilusão pelo trabalho, 43 sujeitos (16,3\%) obtiveram altos níveis de desgaste psíquico, 35 (13,3\%) de indolência e 68 sujeitos $(25,9 \%)$ reportaram altos níveis de culpa.

Em relação aos perfis, dos 263 participantes, 63 (24\%) obtiveram altos níveis do perfil 1, e 29 participantes $(11 \%)$ do perfil 2 , considerado um perfil de adoecimento mais severo. Esses resultados corroboram parcialmente os achados de outras pesquisas. O estudo realizado por Figueiredo-Ferraz, Gil-Monte e GrauAlberola (2009) indicou que 9,0\% dos 211 professores portugueses reportaram níveis baixos de ilusão pelo trabalho, $45,5 \%$ níveis altos de desgaste psíquico, 12,8\% de indolência e $7,1 \%$ de culpa. Nessa pesquisa, trinta $(14,20 \%)$ participantes $(n=211)$ apresentavam níveis elevados de SB (perfil 1) e quatro (1,90\%) relataram níveis mais severos de $\mathrm{SB}$, isto é, equivalentes ao perfil 2.

O estudo de Silva, Pérez, Gonzalez e Ratto (2015) com 71 professores uruguaios demonstrou que a dimensão com maior porcentagem foi a de desgaste psíquico (com 56,4\%), seguida da indolência (45,5\%) e da culpa (31\%), e 30,6\% revelaram baixos índices na ilusão pelo trabalho. Da amostra total, $21,4 \%$ revelaram a presença da SB (perfil l) e 1,4\% evidenciaram altos níveis de culpa (perfil 2). Em contraste, Roa e Dulčić (2018) verificaram que $34 \%$ dos 212 professores chilenos envolvidos no estudo sinalizaram níveis baixos na ilusão pelo trabalho, $48,1 \%$ apresentaram altos níveis em desgaste psíquico, 29,2\% em indolência e $34 \%$ em culpa; com taxa de prevalência da SB de 58\% para o perfil 1 e $25,2 \%$ para o perfil 2 . 
O estudo de Vilela (2014), com uma amostra de 253 professores peruanos, concluiu que 19\% dos participantes apresentaram níveis altos em desgaste psíquico, $10 \%$ em indolência e $49 \%$ em culpa, e nenhum deles revelou níveis baixos na dimensão de ilusão pelo trabalho. As taxas de prevalências da SB foram de $2,4 \%$ e 9,5\% para os perfis 1 e 2 , respectivamente. A pesquisa de Rojas (2010) com 698 professores do México identificou um percentual de $3 \%$ com baixos níveis em ilusão pelo trabalho, $37,4 \%$ com pontuações altas em desgaste psíquico, $4,70 \%$ com altos níveis de indolência e $9,70 \%$ para culpa. Outro estudo mexicano, realizado por Cortés e González (2009), revelou que 2,6\% dos 659 professores estudados apresentaram níveis altos da doença (perfil 1) e 1,1\% apresentaram níveis severos (perfil 2). Ainda no mesmo país, Rojas, Ocaña e Gil-Monte (2008) identificaram níveis da SB relativamente elevados entre 698 professores: $35,5 \%$ (perfil 1) e 17,2\% (perfil 2).

No Brasil, a pesquisa realizada por Gil-Monte, Carlotto e Câmara (2011) apontou que 10,4\% dos 714 participantes revelaram níveis baixos de ilusão pelo trabalho, 36,7\% apresentaram níveis altos de desgaste psíquico, $12,5 \%$ de indolência e $16,1 \%$ de culpa. Nessa investigação, $12 \%$ dos participantes se enquadravam no perfil 1 e 5,6\% no perfil 2. Já os resultados de Carlotto e Câmara (2019) indicaram que 7,5\% dos 679 professores brasileiros se enquadravam no perfil $1 \mathrm{e}$ outros $18,3 \%$ no perfil 2.

Analisando comparativamente os níveis da SB diagnosticados neste estudo e em outros já mencionados, verifica-se que os professores moçambicanos estudados apresentaram maior vulnerabilidade à SB. A docência constitui uma das categorias profissionais mais propensas a desenvolver problemas de saúde mental e com maior prevalência da SB, tal como ilustram estudos feitos em vários países do mundo (Cardoso, Baptista, Sousa, \& Goulart, 2017;
Droogenbroeck \& Spruyt, 2015; Gonçales \& Gonçales, 2016; Roa \& Dulčić, 2018; Rojas et al., 2008; Yang, You, Zhang, Lian, \& Feng, 2019). Por se tratar de uma doença profissional pouco conhecida em Moçambique e que ainda carece de atenção em termos de diagnóstico, prevenção e tratamento, a situação se torna ainda mais preocupante, uma vez que alguns professores investigados revelaram sinais da doença e seguem trabalhando, com risco de agravar o quadro.

\section{Correlações das dimensões da SB com as variáveis sociodemográficas e laborais quantitativas}

Foram realizados testes de correlação de Spearman entre as variáveis quantitativas: idade, número de filhos, anos de serviço, número de alunos para cada professor e carga horária semanal em relação às dimensões de ilusão pelo trabalho, desgaste psíquico, indolência e culpa, conforme apresentado na Tabela 2.

Observa-se, na Tabela 2, que não houve correlações estatisticamente significativas entre as dimensões da SB e as variáveis sociodemográficas e laborais quantitativas, exceto a variável idade com a dimensão de desgaste psíquico, em que a correlação foi significativa, negativa e fraca, com uma proporção de variância de apenas $1,5 \%$, isto é, $1,5 \%$ da variabilidade das respostas da dimensão. Assim, quanto maior a idade, menor o sentimento de desgaste psicológico. Pode-se pensar que essa relação está ligada ao fato de que a idade e o tempo de serviço propiciam maior experiência para lidar com situações estressantes e domínio do próprio ofício. Em consonância, Baptista, Soares, Raad e Santos (2019) observaram que o desgaste psíquico se correlacionou negativamente com idade. Em Rojas et al. (2008), os níveis altos se deram nos sujeitos com maior idade (41 a 50 anos). Desse modo, verificam-se diferenças na relação entre a SB e a variável idade.

Tabela 2

Correlação das variáveis sociodemográficas e laborais quantitativas com as dimensões da SB.

\begin{tabular}{lcccc}
\hline \multicolumn{1}{c}{ Variáveis } & Dlusão pelo trabalho & Desgaste psíquico & Indolência & Culpa \\
Idade & $-0,013$ & $-0,122^{*}$ & 0,024 & $-0,051$ \\
Número de filhos & $-0,045$ & $-0,053$ & 0,046 & $-0,051$ \\
Anos de serviço & 0,030 & $-0,110$ & 0,027 & $-0,084$ \\
Número de alunos & 0,0296 & $-0,0044$ & 0,0433 & 0,0250 \\
Carga horaria semanal & $-0,0311$ & $-0,0300$ & $-0,0049$ & 0,0336 \\
\hline
\end{tabular}

${ }^{*} \mathrm{p}<0,05$ 


\section{Associações das dimensões da SB com as variáveis sociodemográficas e laborais qualitativas}

Para verificar a associação das dimensões da SB com as variáveis sociodemográficas e laborais qualitativas, foi realizado um teste entre elas e as quatro dimensões de CESQT-PE. Os resultados constam na Tabela 3.

A análise dos resultados da Tabela 3 permite observar que apenas a variável sexo teve influência na subescala de culpa, isto é, os professores do sexo masculino denotaram maior presença do sentimento de culpa. Os trabalhos de Carlotto, Braun, Rodriguez e Diehl (2014) e Roa e Dulčić (2018) também demonstraram que participantes do sexo masculino revelaram maior frequência de índices da SB na dimensão de culpa.

Os resultados alcançados neste estudo são preocupantes, uma vez que alguns participantes sinalizaram a presença da SB. Por se tratar de um problema em desenvolvimento, pode ocasionar a debilitação da saúde física e mental do professor e, por conseguinte, comprometer as práticas pedagógicas e a eficácia profissional.

Tabela 3

Associação entre as variáveis sociodemográficas e laborais qualitativas com as dimensões de SB.

\begin{tabular}{|c|c|c|c|c|c|c|c|c|}
\hline \multirow{3}{*}{ Variável categórica } & \multicolumn{8}{|c|}{ Dimensões } \\
\hline & \multicolumn{2}{|c|}{$\begin{array}{l}\text { Ilusão pelo } \\
\text { trabalho }\end{array}$} & \multicolumn{2}{|c|}{ Desgaste psíquico } & \multicolumn{2}{|c|}{ Indolência } & \multicolumn{2}{|c|}{ Culpa } \\
\hline & NA & $\mathrm{BN}$ & NA & $\mathrm{BN}$ & AN & $\mathrm{BN}$ & NA & $\mathrm{BN}$ \\
\hline \multicolumn{9}{|l|}{ Sexo } \\
\hline Masculino & $\begin{array}{c}102 \\
(88,7 \%)\end{array}$ & $\begin{array}{c}13 \\
(11,3 \%)\end{array}$ & $\begin{array}{c}18 \\
(15,7 \%)\end{array}$ & $\begin{array}{c}97 \\
(84,3 \%)\end{array}$ & $15(13 \%)$ & $\begin{array}{c}100 \\
(87 \%)\end{array}$ & $\begin{array}{c}42 \\
(36,5 \%)\end{array}$ & $\begin{array}{c}73 \\
(63,5 \%)\end{array}$ \\
\hline \multirow[t]{2}{*}{ Feminino } & $\begin{array}{c}126 \\
(85,1 \%)\end{array}$ & $\begin{array}{c}22 \\
(14,9 \%)\end{array}$ & $\begin{array}{c}25 \\
(16,9 \%)\end{array}$ & $\begin{array}{c}123 \\
(83,1 \%)\end{array}$ & $\begin{array}{c}20 \\
(13,5 \%)\end{array}$ & $\begin{array}{c}128 \\
(86,5 \%)\end{array}$ & $\begin{array}{c}26 \\
(17,6 \%)\end{array}$ & $\begin{array}{c}122 \\
(82,4 \%)\end{array}$ \\
\hline & \multicolumn{2}{|c|}{$\begin{array}{c}\chi^{2}(1)=0,711 \\
p=0,399\end{array}$} & \multicolumn{2}{|c|}{$\begin{array}{c}\chi^{2}(1)=0,073 \\
p=0,789\end{array}$} & \multicolumn{2}{|c|}{$\begin{array}{c}\chi^{2}(1)=0,012 \\
p=0,911\end{array}$} & \multicolumn{2}{|c|}{$\begin{array}{c}\chi^{2}(1)=12,1 \\
p=0,000\end{array}$} \\
\hline \multicolumn{9}{|l|}{ Situação conjugal } \\
\hline Com companheiro & $\begin{array}{c}170 \\
(85,9 \%)\end{array}$ & $\begin{array}{c}28 \\
(14,1 \%)\end{array}$ & $\begin{array}{c}31 \\
(15,7 \%)\end{array}$ & $\begin{array}{c}167 \\
(84,3 \%)\end{array}$ & $\begin{array}{c}27 \\
(13,6 \%)\end{array}$ & $\begin{array}{c}171 \\
(86,4 \%)\end{array}$ & $\begin{array}{c}53 \\
(26,8 \%)\end{array}$ & $\begin{array}{c}145 \\
(73,2 \%)\end{array}$ \\
\hline \multirow[t]{2}{*}{ Sem companheiro } & $\begin{array}{c}58 \\
(89,2 \%)\end{array}$ & $7(10,8 \%)$ & $\begin{array}{c}12 \\
(18.5 \%)\end{array}$ & $\begin{array}{c}53 \\
(81.5 \%)\end{array}$ & $8(12,3 \%)$ & $\begin{array}{c}57 \\
(87,7 \%)\end{array}$ & $\begin{array}{c}15 \\
(23,1 \%)\end{array}$ & $\begin{array}{c}50 \\
(76,9 \%)\end{array}$ \\
\hline & \multicolumn{2}{|c|}{$\begin{array}{c}\chi^{2}(1)=0,482 \\
p=0,487\end{array}$} & \multicolumn{2}{|c|}{$\begin{array}{c}\chi^{2}(1)=0,282 \\
p=0,596\end{array}$} & \multicolumn{2}{|c|}{$\begin{array}{c}\chi^{2}(1)=0,075 \\
p=0,784\end{array}$} & \multicolumn{2}{|c|}{$\begin{array}{c}\chi^{2}(1)=0,348 \\
p=0,555\end{array}$} \\
\hline \multicolumn{9}{|l|}{ Tipo de vínculo } \\
\hline Nomeado & $\begin{array}{c}208 \\
(87 \%)\end{array}$ & $31(13 \%)$ & $\begin{array}{c}39 \\
(16,3 \%)\end{array}$ & $\begin{array}{c}200 \\
(83,7 \%)\end{array}$ & $\begin{array}{c}33 \\
(13,8 \%)\end{array}$ & $\begin{array}{c}206 \\
(86,2 \%)\end{array}$ & $\begin{array}{c}64 \\
(26,8 \%)\end{array}$ & $\begin{array}{c}175 \\
(73,2 \%)\end{array}$ \\
\hline \multirow[t]{2}{*}{ Contratado } & $\begin{array}{c}20 \\
(83,3 \%)\end{array}$ & $4(16,7 \%)$ & $4(16,7 \%)$ & $\begin{array}{c}20 \\
(83,3 \%)\end{array}$ & $2(8,3 \%)$ & $\begin{array}{c}22 \\
(91,7 \%)\end{array}$ & $4(16,7 \%)$ & $\begin{array}{c}20 \\
(83,3 \%)\end{array}$ \\
\hline & \multicolumn{2}{|c|}{$\begin{array}{c}\chi^{2}(1)=0,037 \\
p=0,847\end{array}$} & \multicolumn{2}{|c|}{$\begin{array}{c}\chi^{2}(1)=0,000 \\
p=1,00\end{array}$} & \multicolumn{2}{|c|}{$\begin{array}{c}\chi^{2}(1)=0,191 \\
p=0,662\end{array}$} & \multicolumn{2}{|c|}{$\begin{array}{l}\chi^{2}(1)=1,16 \\
p=0,281\end{array}$} \\
\hline \multicolumn{9}{|l|}{ Modelo de formação } \\
\hline Básico & $\begin{array}{c}131 \\
(85,1 \%)\end{array}$ & $\begin{array}{c}23 \\
(14,9 \%)\end{array}$ & $\begin{array}{c}29 \\
(18,8 \%)\end{array}$ & $\begin{array}{c}125 \\
(81,2 \%)\end{array}$ & $\begin{array}{c}19 \\
(12,3 \%)\end{array}$ & $\begin{array}{c}135 \\
(87,7 \%)\end{array}$ & $\begin{array}{c}44 \\
(28,6 \%)\end{array}$ & $\begin{array}{c}110 \\
(71,4 \%)\end{array}$ \\
\hline \multirow[t]{2}{*}{ Médio } & $\begin{array}{c}91 \\
(88,3 \%)\end{array}$ & $\begin{array}{c}12 \\
(11,7 \%)\end{array}$ & $\begin{array}{c}14 \\
(13,6 \%)\end{array}$ & $\begin{array}{c}89 \\
(86,4 \%)\end{array}$ & $\begin{array}{c}14 \\
(13,6 \%)\end{array}$ & $\begin{array}{c}89 \\
(86,4 \%)\end{array}$ & $\begin{array}{c}23 \\
(22,3 \%)\end{array}$ & $\begin{array}{c}80 \\
(77,7 \%)\end{array}$ \\
\hline & \multicolumn{2}{|c|}{$\begin{array}{c}\chi^{2}(1)=0,566 \\
p=0,452\end{array}$} & \multicolumn{2}{|c|}{$\begin{array}{c}\chi^{2}(1)=1,21 \\
p=0,270\end{array}$} & \multicolumn{2}{|c|}{$\begin{array}{c}\chi^{2}(1)=0,087 \\
p=0,768\end{array}$} & \multicolumn{2}{|c|}{$\begin{array}{c}\chi^{2}(1)=1,24 \\
p=0,264\end{array}$} \\
\hline
\end{tabular}




\begin{tabular}{|c|c|c|c|c|c|c|c|c|}
\hline \multirow{3}{*}{ Variável categórica } & \multicolumn{8}{|c|}{ Dimensões } \\
\hline & \multicolumn{2}{|c|}{$\begin{array}{l}\text { Ilusão pelo } \\
\text { trabalho }\end{array}$} & \multicolumn{2}{|c|}{ Desgaste psíquico } & \multicolumn{2}{|c|}{ Indolência } & \multicolumn{2}{|c|}{ Culpa } \\
\hline & NA & $\mathrm{BN}$ & NA & $\mathrm{BN}$ & AN & $\mathrm{BN}$ & NA & $\mathrm{BN}$ \\
\hline \multicolumn{9}{|l|}{ Habilitações literárias } \\
\hline Básico & $\begin{array}{c}19 \\
(82,6 \%)\end{array}$ & $4(17,4 \%)$ & $1(4,3 \%)$ & $\begin{array}{c}22 \\
(95,7 \%)\end{array}$ & $3(13,0 \%)$ & $\begin{array}{c}20 \\
(87,0 \%)\end{array}$ & $4(17,4 \%)$ & $\begin{array}{c}19 \\
(82,6 \%)\end{array}$ \\
\hline Médio & $\begin{array}{c}187 \\
(86,6 \%)\end{array}$ & $\begin{array}{c}29 \\
(13,4 \%)\end{array}$ & $\begin{array}{c}39 \\
(18,1 \%)\end{array}$ & $\begin{array}{c}177 \\
(81,9 \%)\end{array}$ & $28(13 \%)$ & $\begin{array}{c}188 \\
(87 \%)\end{array}$ & $\begin{array}{c}61 \\
(28,2 \%)\end{array}$ & $\begin{array}{c}155 \\
(71,8 \%)\end{array}$ \\
\hline \multirow[t]{2}{*}{ Superior } & $\begin{array}{c}22 \\
(91,7 \%)\end{array}$ & $2(8,3 \%)$ & $3(12,5 \%)$ & $\begin{array}{c}21 \\
(87,5 \%)\end{array}$ & $4(16,7 \%)$ & $\begin{array}{c}20 \\
(83,3 \%)\end{array}$ & $3(12,5 \%)$ & $\begin{array}{c}21 \\
(87,5 \%)\end{array}$ \\
\hline & \multicolumn{2}{|c|}{$\begin{array}{c}\chi^{2}(2)=0,850 \\
p=0,654\end{array}$} & \multicolumn{2}{|c|}{$\begin{array}{c}\chi^{2}(2)=3,14 \\
p=0,208\end{array}$} & \multicolumn{2}{|c|}{$\begin{array}{c}\chi^{2}(2)=0,258 \\
p=0,879\end{array}$} & \multicolumn{2}{|c|}{$\begin{array}{c}\chi^{2}(2)=3,73 \\
p=0,155\end{array}$} \\
\hline \multicolumn{9}{|l|}{ Carreira/Categoria } \\
\hline Básico/DN4 & $\begin{array}{c}89 \\
(84,8 \%)\end{array}$ & $\begin{array}{c}16 \\
(15,2 \%)\end{array}$ & $\begin{array}{c}18 \\
(17,1 \%)\end{array}$ & $\begin{array}{c}87 \\
(82,9 \%)\end{array}$ & $\begin{array}{c}13 \\
(12,4 \%)\end{array}$ & $\begin{array}{c}92 \\
(87,6 \%)\end{array}$ & $\begin{array}{c}33 \\
(31,4 \%)\end{array}$ & $\begin{array}{c}72 \\
(68,6 \%)\end{array}$ \\
\hline Médio/DN3 & $\begin{array}{c}129 \\
(87,8 \%)\end{array}$ & $\begin{array}{c}18 \\
(12,2 \%)\end{array}$ & $25(17 \%)$ & $\begin{array}{c}122 \\
(83 \%)\end{array}$ & $\begin{array}{c}19 \\
(12,9 \%)\end{array}$ & $\begin{array}{c}128 \\
(87,1 \%)\end{array}$ & $\begin{array}{c}34 \\
(23,1 \%)\end{array}$ & $\begin{array}{c}113 \\
(76,9 \%)\end{array}$ \\
\hline \multirow[t]{2}{*}{ Superior/DN2 e N1 } & $\begin{array}{c}10 \\
(90,9 \%)\end{array}$ & $1(9,1 \%)$ & $0(0,0 \%)$ & $\begin{array}{c}11 \\
(100 \%)\end{array}$ & $3(27,3 \%)$ & $8(72,7 \%)$ & $1(9,1 \%)$ & $\begin{array}{c}10 \\
(90,9 \%)\end{array}$ \\
\hline & \multicolumn{2}{|c|}{$\begin{array}{c}\chi^{2}(2)=0,653 \\
p=0,722\end{array}$} & \multicolumn{2}{|c|}{$\begin{array}{c}\chi^{2}(2)=2,25 ; \\
p=0,326\end{array}$} & \multicolumn{2}{|c|}{$\begin{array}{c}\chi^{2}(2)=1,96 \\
p=0,376\end{array}$} & \multicolumn{2}{|c|}{$\begin{array}{c}\chi^{2}(2)=3,88 \\
p=0,143\end{array}$} \\
\hline
\end{tabular}

AN = Alto nível; BN = Baixo nível.

\section{Considerações finais}

Este estudo avaliou a frequência da SB em professores moçambicanos do ensino fundamental, verificando as possíveis associações com as variáveis sociodemográficas e laborais. O principal mérito deste estudo é ser pioneiro na pesquisa sobre a SB neste segmento da população com o uso do instrumento CESQT-PE. Além disso, representa um avanço importante na compreensão do constructo estudado no contexto de trabalho dos professores moçambicanos do ensino básico, pois constitui uma contribuição para a limitada literatura sobre saúde mental e qualidade de vida profissional desse grupo.

Em função dos resultados, depreende-se que alguns professores inquiridos apresentaram maior risco de desenvolver a $\mathrm{SB}$, que se caracteriza pela frequência de sentimentos nas quatro dimensões do modelo usado para avaliação do constructo, com destaque para as dimensões de desgaste psíquico e culpa. Os resultados se mostram preocupantes pelo fato de quase um quarto (24\%) dos participantes se enquadrarem no perfil 1 (baixos níveis em ilusão pelo trabalho, altos níveis em desgaste psíquico e indolência) e
$11 \%$ serem acompanhados pelo sentimento de culpa (perfil 2). É fundamental levar em consideração tais resultados, pois o modelo adotado neste estudo considera que a presença do sentimento de culpa indica níveis severos da síndrome. Ademais, os achados da pesquisa sustentam que a SB está relacionada a características pessoais específicas, conforme demonstrado pela correlação estatisticamente significativa entre as variáveis idade e sexo e as dimensões de desgaste psíquico e culpa, respectivamente.

Os resultados chamam a atenção dos gestores educacionais e da comunidade escolar para a necessidade de melhoria dos fatores geradores de estresse ocupacional, contemplando a reestruturação das formas de organização do trabalho docente, de modo a transformá-lo em saudável e prazeroso. Além do mais, é importante desenvolver ações de capacitação e formação continuada dos professores em matéria de gestão de estresse, de modo a despertar atitudes de boas práticas de saúde mental como forma de conscientização para que consigam lidar com diferentes obrigações e situações estressantes de forma mais adequada, com vista a promover o bem-estar dos 
profissionais. Igualmente, a pesquisa sugere o delineamento e implantação de serviços de atenção à saúde do trabalhador (e.g., professor) moçambicano em todos os níveis (nacional, provincial e distrital) para o devido acompanhamento psicossocial e capacitação.

Este estudo possui algumas limitações que devem ser consideradas na análise de seus resultados e conclusões. A primeira tem a ver com sua natureza transversal, que impede conclusões em termos de causalidade. A segunda está relacionada à regionalidade e à cultura em que a população da amostra não probabilística respondente se insere, bem como ser composta apenas por professores da cidade de Nampula, podendo não ser passível a generalização e a inserção em outras culturas. Mesmo assim, os resultados alcançados servem de indicativos para melhor compreensão da SB na população pesquisada. Em face dessas limitações, afigura-se importante a realização de futuras pesquisas envolvendo amostras probabilísticas, bem como estudos comparativos entre professores das zonas rurais e urbanas, ou mesmo de diferentes níveis do ensino, visando uma melhor compreensão da SB em Moçambique que possam fundamentar programas de prevenção e intervenção.

\section{Referências}

Abacar, M. (2011). Stress ocupacional e o bem-estar de professores do ensino básico em escolas moçambicanas [Dissertação de mestrado, Universidade do Porto]. Repositório da Universidade do Porto. https://repositorioaberto.up.pt/handle/10216/114560

Abacar, M. (2015). Burnout em docentes do ensino básico em escolas moçambicanas e brasileiras [Tese de doutorado, Universidade Federal de Pernambuco]. Repositório da Universidade Federal de Pernambuco. https:// repositorio. ufpe.br/bitstream/123456789/17388/1/Tese\%20\%20Mussa\%20-\%20\%2021.01.2015.pdf

Abacar, M., Aliante, G., \& António, F. (2020). Burnout em professores do ensino secundário. Research, Society and Development, 9(7), 1-25. http://dx.doi.org/10.33448/rsd-v9i7.3776

Abacar, M., Aliante, G., \& Nahia, I. A. A. (2020). Fontes de estresse ocupacional e estratégias de enfrentamento em professores moçambicanos do ensino básico. Saúde e Pesquisa, 13(1), 41-52. https://doi.org/ 10.17765/2176-9206.2020v13n1p41-52

Abacar, M., Roazzi, A., \& Bueno, J. M. H. (2017). Estresse ocupacional: Percepções dos professores. Revista Amazônica, 19(1), 430-472.

Abacar, M., Tarcísio, L., \& Aliante, G. (2017). Burnout em professores moçambicanos do ensino superior público e privado. Saúde e Pesquisa, 10(3), 567-577. https://doi.org/10.17765/2176-9206.2017v10n3p567-577

Aliante, G. (2018). Síndrome de burnout e trabalho: Um estudo junto a professores moçambicanos do ensino fundamental das escolas da rede pública na cidade de Nampula [Dissertação de mestrado, Instituto de Psicologia da Universidade Federal do Rio Grande do Sul]. Lume Repositório Digital. http://hdl.handle.net/10183/187611

Aliante, G., Abacar, M., \& Pereira, A. M. (2020). Estresse ocupacional em professores de educação inclusiva. Estudos Interdisciplinares em Psicologia, 11(1), 162-181. https://doi.org/10.5433/2236-6407.2020v11nlp162

Aliante, G., \& Tittoni. (2017, 1-4 de novembro). Saúde mental em Moçambique: Percepção dos professores do ensino fundamental da rede pública de angoche sobre os fatores psicossociais de risco no trabalho docente. Encontro Nacional ABRAPSO: Democracia participativa, Estado e Laicidade, Uberlândia, MG, Brasil.

Amimo, C. A. (2012). Are you experiencing teacher burnout? A synthesis of research reveals conventional prevention and spiritual healing. Education Research Journal, 2(11), 338-344.

Antoniou, A-S., Ploumpi, A., \& Ntalla, M. (2013). Occupational stress and professional burnout in teachers of primary and secondary education: The role of coping strategies. Psychology, 4(3), 349-355. http://dx.doi.org/ 10.4236/psych.2013.43A051

Badawy, S. M. (2015). Egyptian teachers' burnout: The role of work environment characteristics and job stress. Journal of Business and Management Sciences, 3(4), 101-110.

Baptista, M. N., Soares, T. F. P., Raad, A. J., \& Santos, L. M. (2019). Burnout, estresse, depressão e suporte laboral em professores universitários. Revista Psicologia: Organizações e Trabalho, 19(1), 564-570. http://dx.doi.org/ $10.17652 / \mathrm{rpot} / 2019.1 .15417$ 
Cardoso, H. F., Baptista, M. N., Sousa, D. F. A., \& Goulart Jr., E. (2017). Síndrome de burnout: Análise da literatura nacional entre 2006 e 2015. Revista Psicologia: Organizações e Trabalho, 17(2), 121-128. http://dx.doi.org/ $10.17652 / \mathrm{rpot} / 2017.2 .12796$

Carlotto, M. S., Braun, A. C., Rodriguez, S. Y. S., \& Diehl, L. (2014). Burnout em professores: Diferença e análise de gênero. Contextos Clínicos, 7(1), 86-93. http://dx.doi.org/10.4013/ctc.2014.71.08

Carlotto, M. S., \& Câmara, S. G. (2019). Prevalence and predictors of burnout syndrome among public elementary school teachers. Análise Psicológica, 37(2), 135-146. http://dx.doi.org/10.14417/ap.1471

Carlotto, M. S., Câmara, S. G., Diehl, L., Ely, K., Freitas, I. M., \& Schneider, G. A. (2018). Estressores ocupacionais e estratégias de enfrentamento. Revista Subjetividades, 18(1), 92-105. http://dx.doi.org/10.5020/23590777. rs.v18i1.6462

Cortés, V. S. N., \& González, L. E. P. (2009). Desgaste profesional (Síndrome Burnout) en profesores de escuelas primarias públicas del municipio de Aguascalientes. X Congreso Nacional de Investigación Educativa, Veracruz, Espanha. https://www.uv.es/unipsico/pdf/CESQT/Externos/2009_Noyola_y_Padilla.pdf

Cruz, R. M., Lemos, J. C., Welter, M. M., \& Guisso, L. (2010). Saúde docente, condições e carga de trabalho. Revista Electrónica de Investigación y Docencia, (4), 147-160.

Droogenbroeck, F. V., \& Spruyt, B. (2015). Do teachers have worse mental health? Review of the existing comparative research and results from the Belgian Health Interview Survey. Teaching and Teacher Education, 51, 88-100. https://doi.org/10.1016/j.tate.2015.06.006

Figueiredo-Ferraz, H., Gil-Monte, P. R., Grau-Alberola, E. (2009). Prevalencia del síndrome de quemarse por el trabajo (burnout) en una muestra de maestros portugueses. Aletheia, 29, 6-15.

Figueiredo-Ferraz, H., Gil-Monte, P. R., \& Grau-Alberola, E. (2013). Psychometric properties of the "Spanish Burnout Inventory" (SBI): Adaptation and validation in a Portuguese-speaking sample. European Review of Applied Psychology, 63(1), 33-40. https://doi.org/10.1016/j.erap.2012.08.003

Genoud, P. A., Brodard, F., \& Reicherts, M. (2009). Facteurs de stress et burnout chez les enseignants de l'écoleprimaire. European Review of Applied Psychology, 59(1), 37-45. https://doi.org/10.1016/j.erap.2007.03.001

Gil-Monte, P. R. (2005). El síndrome de quemarse por el trabajo (burnout): Una enfermedad laboral en la sociedad del bienestar. Psicología Pirámide.

Gil-Monte, P. R. (2008). El síndrome de quemarse por el trabajo (burnout) como fenómeno transcultural. Informació Psicològica, (91-92), 4-11.

Gil-Monte, P. R., Carlotto, M. S., \& Câmara, S. G. (2010). Validação da versão brasileira do "Cuestionario para la EvaluacióndelSíndromedeQuemarseporelTrabajo” emprofessores.SaúdePública, 44(1), 140-147.https://doi.org/ $10.1590 /$ S0034-89102010000100015

Gil-Monte, P. R., Carlotto, M. S., \& Câmara, S. G. (2011). Prevalence of burnout in a sample of Brazilian teachers. The European Journal of. Psychiatry, 25(4), 205-212. http://dx.doi.org/10.4321/S0213-61632011000400003

Gomes, A. R., Peixoto, A., Pacheco, R., \& Silva, M. (2012). Stress ocupacional e alteração do Estatuto da Carreira Docente português. Educação e Pesquisa, 38(2), 357-371. https://doi.org/10.1590/S1517-97022012005000008

Gonçales, C. A., \& Gonçales, R. A. (2016). Síndrome de burnout: Causas e consequências em diversos profissionais. Revista Brasileira de Psicologia, 3(2), 49-65.

Guglielmi, R. S., \& Tatrow, K. (1998). Occupational stress, burnout, and health in teachers: A methodological and theoretical analysis. Review of Educational Research, 68(1), 61-69. https://doi.org/10.3102\%2F00346543068001061

INDE/MINED. Moçambique. (2003). Plano Curricular do Ensino Básico.

Lambert, E. G., Hogan, N. L., Griffin, M. L., \& Kelley, T. (2015). The correctional staff burnout literature. Criminal Justice Studies, 28(4), 397-443. https://doi.org/10.1080/1478601X.2015.1065830

Lhospital, A. S., \& Gregory, A. (2009). Changes in teacher stress through participation in prereferral intervention teams. Psychology in the Schools, 46(10), 1098-1112.

Maslach, C., \& Goldberg, J. (1998). Prevention of burnout: New perspective. Applied and Preventive Psychology, 7(1), 63-74. https://psycnet.apa.org/doi/10.1016/S0962-1849(98)80022-X

Maslach, C., \& Jackson, S. (1981). The measurement of experienced burnout. Journal of Occupational Behaviour, 2(2), 99-113. https://doi.org/10.1002/job.4030020205 
Maslach, C., \& Leiter, M. P. (2017). New insights into burnout and health care: Strategies for improving civility and alleviating burnout. Medical Teacher, 39(2), 160-163. https://doi.org/10.1080/0142159X.2016.1248918

MINED. (2015). Relatório sobre os seis objectivos da educação para todos.

Olaitan, O. L., Oyerinde, O. O., Obiyemi, O., \& Kayode, O. O. (2010). Prevalence of job stress among primary school teachers in South West, Nigeria. African Journal of Microbiology Research, 4(5), 339-342.

Oliveira, H. L. R., Balk, R. S., Graup, S., \& Muniz, A. G. (2020). Percepções sobre saúde mental de professores e professoras de uma escola pública da fronteira oeste do Rio Grande do Sul. Research, Society and Development, 9(4), 1-16. http://dx.doi.org/10.33448/rsd-v9i4.3060

Pocinho, M., \& Capelo, M. R. (2009). Vulnerabilidade ao stress, estratégias de coping e autoeficácia em professores portugueses. Educação e Pesquisa, 35(2), 351-367. https://doi.org/10.1590/S1517-97022009000200009

Roa, J. A. S., \& Dulčić, F. J. L. (2018). Síndrome de burnout y calidad de vida profesional percibida según estilos de personalidad en profesores de educación primaria. CES Psicología, 11(1), 69-89. http://dx.doi.org/ $10.21615 /$ cesp.11.1.6

Rojas, S. U. (2010). Estudio de prevalencia del síndrome de quemarse por el trabajo (SQT) en maestros de primaria de la ciudad de México y su asociación con sobrecarga y autoeficacia. Ciencia \& Trabajo, 35, 257-262.

Rojas, S. U., Ocaña, J. I. S., \& GilMonte, P. R. (2008). Prevalencia del síndrome de quemarse por el trabajo (SQT) (burnout) en maestros mexicanos. Informació Psicològica, 91-92, 53-63.

Salvagioni, D. A. J., Melanda, F. N., Mesas, A. E., González, A. D., Gabani, F. L., \& Andrade, S. M. (2017). Physical, psychological and occupational consequences of job burnout: A systematic review of prospective studies. PLOS ONE, 12(10), 1-29. https://doi.org/10.1371/journal.pone.0185781

Schaufeli,W., \& Enzmann, D. (1998). The burnout companion to study and practice:A critical analysis. Taylor \& Francis.

Silva, M. I., Pérez, R. C. G., González, M. G., \& Ratto, A. (2015). Prevalencia del síndrome de quemarse por el trabajo y variables sociodemográficas en un grupo de maestras de Montevideo. Ciencias Psicológicas, 9(1), 55-62.

Vasconcelos, A. C. L., \& Neves, M. Y. (2010). A saúde de professoras do ensino fundamental: Relato de uma investigação. In M. Y. Neves, A. J. S. Araujo, S. B. Vieira, \& M. F. P. Alberto (Orgs.), Subjetividade e trabalho: A vida não é só isso que se vê(pp. 92-110). Educar.

Vilela, Y. E. B. (2014). Factores asociados al burnout en docentes de secundaria de colegios públicos [Monografia, Pontificia Universidad Catolica del Perú]. Repositório da PUCP. http://tesis.pucp.edu.pe/repositorio/ handle/20.500.12404/5550

Yang, R., You, X., Zhang, Y., Lian, L., \& Feng, W. (2019). Teachers' mental health becoming worse: The case of China. International Journal of Educational Development, 70. https://doi.org/10.1016/j.ijedudev.2019.102077

Yong, Z., \& Yue, Y. (2007). Causes for burnout among secondary and elementary school teachers and preventive strategies. Chinese Education and Society, 40(5), 78-85. https://doi.org/10.2753/CED1061-1932400508

Zhuwao, S., Setati, S. T., Rachidi, M. P., \& Ukpere, W. I. (2015). Occupational stress and organisational commitment of employees at higher educational institution. Journal of Governance and Regulation, 4(4), 740-746. https://doi.org/ 10.22495/jgr_v4_i4_c6_p10

\section{Gildo Aliante}

Mestre e doutorando, com bolsa da Capes, em Psicologia Social e Institucional pelo Instituto de Psicologia da Universidade Federal do Rio Grande do Sul (Ufrgs), Porto Alegre - RS. Brasil.

E-mail: aliantegildo@yahoo.com.br

(1) http:// orcid.org/0000-0002-6283-9544

\section{Jaqueline Tittoni}

Professora Adjunta do Programa de Pós-Graduação em Psicologia Social e Institucional do Instituto de Psicologia da Universidade Federal do Rio Grande do Sul (Ufrgs), Porto Alegre - RS. Brasil.

E-mail: jatittoni@gmail.com

(1) https://orcid.org/0000-0002-3450-080X 


\section{Mary Sandra Carlotto}

Professora do Programa de Pós-Graduação em Psicologia na Universidade do Vale do Rio dos Sinos (Unisinos), São Leopoldo - RS. Brasil.

E-mail: mscarlotto@gmail.com

(D) https://orcid.org/0000-0003-2336-5224

\section{Mussa Abacar}

Doutor em Psicologia Cognitiva pela Universidade Federal de Pernambuco (UFPE). Professor da Faculdade de Educação e Psicologia da Universidade Rovuma, Nampula. Moçambique.

E-mail: mabacar@unirovuma.ac.mz

(i) http://orcid.org/0000-0001-7797-8101

Agradecimentos especiais ao PEC-PG/CNPq pelo financiamento de bolsa de mestrado oferecida ao primeiro autor deste trabalho. Também os nossos sinceros agradecimentos vão aos professores moçambicanos do ensino primário na cidade de Nampula que participaram da pesquisa, ao Serviço Distrital de Educação, Juventude e Tecnologia de Nampula pela autorização para a realização da pesquisa, bem como às direções das escolas, direção do Departamento de Ciências de Educação e Psicologia da Universidade Pedagógica de Nampula (atual Faculdade de Educação e Psicologia da Universidade Rovuma), pela contribuição na coleta de dados.

Endereço para envio de correspondência:

Instituto de Psicologia da Ufrgs, Rua Ramiro Barcelos, 2600, Santana. CEP: 90035-003. Porto Alegre - RS. Brasil.

Recebido 13/02/2019

Aceito 20/07/2020

Received 02/13/2019

Approved 07/20/2020

Recibido 13/02/2019

Aceptado 20/07/2020

Como citar: Aliante, G., Tittoni, J., Carlotto, M. S., \& Abacar, M. (2021). Síndrome de burnout em professores moçambicanos do ensino fundamental. Psicologia: Ciência e Profissão, 41, 1-14. https://doi.org/10.1590/ $1982-3703003219900$

How to cite: Aliante, G., Tittoni, J., Carlotto, M. S., \& Abacar, M. (2021). Burnout syndrome in mozambican teachers of elementary school. Psicologia: Ciência e Profissão, 41, 1-pp. https://doi.org/10.1590/1982-3703003219900

Cómo citar: Aliante, G., Tittoni, J., Carlotto, M. S., \& Abacar, M. (2021). Síndrome de quemarse por el trabajo en profesores mozambiqueños de la primaria. Psicologia: Ciência e Profissão, 41, 1-14. https://doi.org/10.1590/ 1982-3703003219900 